\title{
The unclear role of VEGF in POEMS syndrome: therapeutic implications of neoangiogenesis in a rare plasma cell disorder
}

\author{
Gianfranco Lapietra, Francesca Fazio, Maria Teresa Petrucci \\ Hematology, Department of Translational and Precision Medicine, Sapienza University, Rome 00161, Italy. \\ Correspondence to: Prof. Maria Teresa Petrucci, Hematology, Department of Translational and Precision Medicine, Sapienza \\ University of Rome, Via Benevento, 6, Rome 00161, Italy. E-mail: petrucci@bce.uniroma1.it
}

How to cite this article: Lapietra G, Fazio F, Petrucci MT. The unclear role of VEGF in POEMS syndrome: therapeutic implications of neoangiogenesis in a rare plasma cell disorder. J Cancer Metastasis Treat 2021;7:61. https://dx.doi.org/10.20517/23944722.2021 .106

Received: 30 Apr 2021 First Decision: 9 Jun 2021 Revised: 16 Jun 2021 Accepted: 21 Jun 2021 Published: 5 Nov 2021

Academic Editors: Domenico Ribatti, Lucio Miele Copy Editor: Xi-Jun Chen Production Editor: Xi-Jun Chen

\begin{abstract}
POEMS syndrome is a rare paraneoplastic disorder due to an underlying clone of aberrant plasma cells. The name POEMS is an acronym for some of the major disease manifestations, namely polyneuropathy, organomegaly, endocrinopathy, presence of monoclonal component, and skin changes. The clinical presentation can be various and could lead to delayed diagnosis and treatment. Little is known about the pathogenic mechanism, although the neoangiogenesis due to overproduction of vascular endothelial growth factor (VEGF) by plasma cells seems to play a key role. The latest evidence suggests that the blood concentration of this cytokine correlates with the activity of the syndrome: VEGF could then be used as a therapeutic target and a marker to monitor response. Several case reports have shown the efficacy of this approach, but extended studies are required to better define the use of antiVEGF in patients affected by POEMS syndrome.
\end{abstract}

Keywords: POEMS syndrome, VEGF, anti-VEGF, plasma cell disorder

\section{INTRODUCTION}

POEMS (polyneuropathy, organomegaly, endocrinopathy, presence of monoclonal component, and skin changes) syndrome is a rare paraneoplastic syndrome due to a clonal plasma cell proliferative disorder. The 
disease can occur with a great variety of signs and symptoms; for this reason, it can be misdiagnosed at the onset and the treatment can be delayed. Some specific diagnostic criteria have been identified to ease the identification. The diagnostic criteria are divided as follows:

Mandatory major criteria:

(1) Polyneuropathy (typically demyelinating);

(2) Monoclonal plasma cell disorder (typically, $\lambda$-light chain restricted).

Other major criteria:

(1) Castleman disease;

(2) Sclerotic bone lesions;

(3) Vascular endothelial growth factor elevation.

Minor criteria:

(1) Organomegaly (mainly splenomegaly and hepatomegaly, sometimes lymphadenopathies);

(2) Extravascular volume overload (ascites and pleural and pericardial effusion);

(3) Endocrinopathy (mainly involving the gonadal axis, sometimes involving the adrenal and the thyroid axes);

(4) Skin changes (especially due to overproduction of melanin);

(5) Papilledema;

(6) Thrombocytosis/polycythemia.

The confirmation of diagnosis requires the coexistence of the mandatory major criteria with at least one of the other major criteria and one of the minor criteria.

The epidemiology of the disease is not well established given its very low incidence ${ }^{[1]}$.

\section{THE MOLECULAR LANDSCAPE}

VEGF is a ubiquitarious heterodimeric glycoprotein belonging to the family of growth factors. Several isoforms have been identified in humans: VEGF-A, VEGF-B, VEGF-C, VEGF-D, VEGF-E, VEGF-F, placenta growth factor, and endocrine gland-derived VEGF (EG-VEGF) ${ }^{[2]}$. They are all involved in the process of vasculogenesis and lymphagenesis during the embryonic period and, after birth, in the process of physiologic and pathological angiogenesis. The signaling is mediated by the stimulation of ubiquitarious VEGF receptors (VEGFR) with an intracellular tyrosine kinase activity. The interaction between VEGF and its receptor plays a leading role in the tumorigenesis: in the pathologic microenvironment, hypoxia, due to the waste of energy by the proliferating cells, stimulates the VEGF/VEGFR binding. This cooperation induces migration of inflammatory cells; the subsequent proangiogenic cytokines storm favors the creation of new aberrant vascular networks, resulting in tumor growth and metastatic dissemination.

The pathogenic mechanism of POEMS syndrome is far from being fully understood, but increased production of VEGF by clonal bone marrow plasma cells could be one of the driving forces, as suggested by a Chinese study ${ }^{[3]}$ [Figure 1]. In this study, serum VEGF levels were measured using an enzyme immunoassay in 62 patients affected by POEMS syndrome, at the onset and after standard treatment with lenalidomide and dexamethasone. VEGF mRNA expression in the bone marrow plasma cells at diagnosis was notably higher than in samples sourced from patients with other plasma cell neoplasms. After induction therapy, the normalization of VEGF serum was observed in $49.2 \%$ of POEMS patients, most of them also achieving a complete hematologic response. The same study revealed in the 62 patients the presence of 


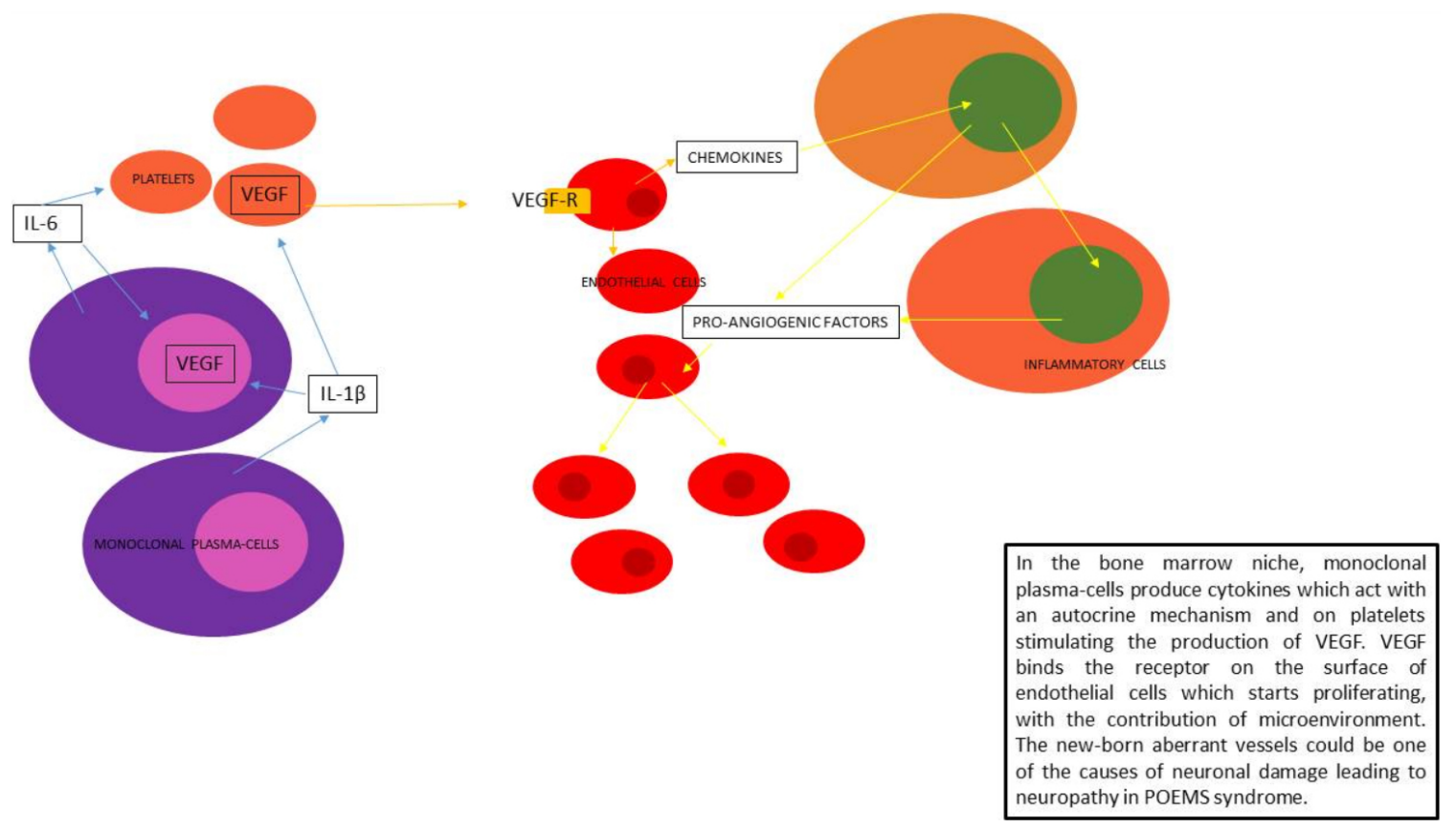

Figure 1. Mechanism of production of VEGF and possible mechanism of action in the pathogenesis of POEMS syndrome. VEGF: Vascular endothelial growth factor; VEGF-R: VEGF receptor.

polyclonal plasma cells, beyond the monoclonal population: even if there is no substantial difference in the intracellular VEGF levels, the monoclonal counterpart produces higher levels of cytokines, such as IL-1 $\beta$ and IL-6. These cytokines are responsible for the overproduction of VEGF. On the other side, Nagao et al. ${ }^{[4]}$ performed targeted sequencing of 20 cases with POEMS searching for possible driver mutations: in all patients, recurrent mutations in seven genes were identified, but surprisingly none of these concerned VEGF genes family.

\section{THE CLINICAL UTILITY VEGF as a surrogate biomarker}

Even if the role of VEGF in the pathogenesis of POEMS remains unclear, it is still the most important cytokine correlating with disease activity. In a large study performed by the Mayo Clinic on 105 patients with different diseases accompanied by overproduction of VEGF (POEMS syndrome, Castleman disease, multiple myeloma, amyloidosis, connective diseases, and vasculitis), higher levels of VEGF were found in patients with POEMS at onset ${ }^{[5]}$. The levels dramatically decreased in patients with a complete response after treatment, with no statistically significant difference among the therapeutic regimens. VEGF levels could be thus used for diagnosing as well as monitoring response in patients on active treatment. The principal isoform of VEGF we can measure both in plasma and in serum samples is VEGF165: to our knowledge, the best cut-offs are 200 and $1920 \mathrm{pg} / \mathrm{mL}$, respectively ${ }^{\left[{ }^{[}\right]}$. Whether the measurement should be made on plasma or serum is still unclear. Serum usually presents with higher levels due to platelet activation during the serum processing with subsequent release of VEGF. The use of this biologic material could then overestimate the in vivo real amount of the cytokine. In 2003, Tokashiki et al. ${ }^{[6]}$ reported a fatal case of disseminated intravascular coagulation in a 62-year-old patient affected by POEMS. The platelet activation caused a massive release of VEGF, which is supposed to play a role in the coagulopathy as well. According to them, the use of serum is the most reliable method since it represents the contribution from both intracellular and extracellular compartments. However, the method has not been standardized yet, and the consensus is far from being reached, also given the rarity of the disease. 
Table 1. Published studies on use of VEGF as surrogate biomarker and published case reports on use of antibodies targeting VEGF in POEMS syndrome

\begin{tabular}{|c|c|}
\hline \multicolumn{2}{|l|}{ VEGF as a surrogate biomarker } \\
\hline Serum VEGF levels are higher in POEMS patients and decreases in responders to front-line standard therapy & Wang et al. ${ }^{[3]}(2016)$ \\
\hline Recurrent genetic mutations are identified in POEMS patients but none of them concerns VEGF genes family & Nagao et al. ${ }^{[4]}$ (2019) \\
\hline \multicolumn{2}{|l|}{ VEGF as a therapeutic target } \\
\hline \multirow[t]{3}{*}{ Monoclonal antibody targeting VEGF as single agent } & Garcia et al. $^{[7]}(2020)$ \\
\hline & Badros et al. ${ }^{[8]}(2005)$ \\
\hline & Straume et al. ${ }^{[10]}(2006)$ \\
\hline \multirow[t]{3}{*}{ Regimens containing monoclonal antibody targeting VEGF for naïve and relapsed/refractory POEMS syndrome } & Dietrich et al. ${ }^{[9]}(2008)$ \\
\hline & Samaras et al. ${ }^{[11]}(2007)$ \\
\hline & Ohwada et al. ${ }^{[12]}(2009)$ \\
\hline
\end{tabular}

VEGF: Vascular endothelial growth factor.

\section{VEGF as a therapeutic target}

The evidence of high levels of VEGF in serum and plasma of patients with POEMS guided the idea that the syndrome could be successfully treated with drugs directed against this cytokine. Bevacizumab is a recombinant humanized monoclonal antibody binding to the circulating VEGF-A isoform, thus blocking its interaction with VEGFR ${ }^{[7]}$. It was first approved in 2004 by regulatory institutions for the treatment of metastatic colon cancer, and since then it has been used in a variety of solid tumors. Very few case reports have shown the efficacy of bevacizumab as single agent in the treatment of POEMS [Table 1]. Badros et al. ${ }^{[8]}$ described the case of a 52-year-old woman with serious POEMS who achieved a complete hematologic response after two months of therapy with melphalan and dexamethasone, without any improvement in the progressive debilitating neuropathy. The compassionate use of bevacizumab resulted in a dramatic improvement of symptoms accompanied by rapid decrease in VEGF levels both in plasma and in serum. A great response to VEGF inhibition was also reported in a 45 -year-old woman with a serious neuropathy at onset: in this case, the reduction of the disease burden thanks to bevacizumab allowed the management of an autologous stem-cell transplant, with a further and lasting improvement of the response $e^{[0]}$. Straume et al. ${ }^{[10]}$ reported the case of a 41 -year-old male with POEMS not responding to conventional therapy with cyclophosphamide. He was given bevacizumab, but he developed a fatal capillary leak syndrome. It could be speculated that the cause was the rapid decrease in VEGF, leading to an excessive permeabilization of the blood-brain barrier, but the data are too limited to give valid considerations. Other authors have explored the combination of bevacizumab with chemotherapy regimens, with contrasting results. In the experience of Samaras et al. ${ }^{[11]}$, the late relapse of POEMS in a 49-year-old man was treated with intravenous cyclophosphamide, oral steroids, and bevacizumab without any improvement in the peripheral neuropathy or the pulmonary hypertension he developed six years after the front-line therapy. On the other side, VEGF was successfully used in combination with thalidomide in a patient affected by POEMS, with cardiac and respiratory distress due to massive pericardial and pleural effusion ${ }^{[12]}$. The rapid improvement of the general status allowed the autologous stem-cell transplant and the complete recovery of the patient. In this case, the depth of the response could also be due to the association with thalidomide: anti-VEGF activity is included in the wide spectrum of action of immunomodulants ${ }^{[13]}$.

\section{CONCLUSION}

POEMS syndrome is a rare paraneoplastic disease with a multiorgan involvement which could result fatal in some cases. The mechanism leading to this syndrome is not known, but a central role seems to be played by VEGF. Despite the pathogenic importance of this cytokine, the attempts to use it as a marker disease and a therapeutic target are anecdotal and often unsatisfactory. Due to the rarity of the disease, the main 
information about its treatment in general is based more on real-life experience than on randomized trials. At our Department of Translational and Precision Medicine, patients with POEMS syndrome have been successfully treated with alkylator-based therapy, followed by autologous stem-cell transplant, according to age and the presence of another comorbidity. Based on the extraordinary results shown in other plasma-cell neoplasms, immunomodulants and proteasome inhibitors, such as lenalidomide and ixazomib, respectively, either alone or in combination with high-dose steroids, and monoclonal antibodies targeting CD38 antigen, such as daratumumab, have been investigated in several clinical trials for patients with POEMS syndrome (NCT01816620, NCT02921893, and NCT04396496). The results from these trials are awaited.

Meanwhile, further efforts are required to better identify the real role of VEGF and clarify whether it can be used in clinical practice.

\section{DECLARATIONS}

\section{Authors' contributions}

Wrote the manuscript: Lapietra G

Revised the manuscript: Fazio F, Petrucci MT

All the named authors have read and approved the submitted manuscript.

\section{Availability of data and materials}

Not applicable.

\section{Financial support and sponsorship}

None.

\section{Conflict of interests}

All authors declared that there are no conflicts of interest.

\section{Ethical approval and consent to participate}

Not applicable.

\section{Consent for publication}

Not applicable.

\section{Copyright}

(c) The Author(s) 2021.

\section{REFERENCES}

1. Dispenzieri A. POEMS Syndrome: 2019 Update on diagnosis, risk-stratification, and management. Am J Hematol 2019;94:812-27. DOI PubMed

2. Melincovici CS, Boşca AB, Şuşman S, et al. Vascular endothelial growth factor (VEGF) - key factor in normal and pathological angiogenesis. Rom J Morphol Embryol 2018;59:455-67. PubMed

3. Wang C, Huang XF, Cai QQ, et al. Remarkable expression of vascular endothelial growth factor in bone marrow plasma cells of patients with POEMS syndrome. Leuk Res 2016;50:78-84. DOI PubMed

4. Nagao Y, Mimura N, Takeda J, et al. Genetic and transcriptional landscape of plasma cells in POEMS syndrome. Leukemia 2019;33:1723-35. DOI PubMed

5. D'Souza A, Hayman SR, Buadi F, et al. The utility of plasma vascular endothelial growth factor levels in the diagnosis and follow-up of patients with POEMS syndrome. Blood 2011;118:4663-5. DOI PubMed

6. Tokashiki T, Hashiguchi T, Arimura K, Eiraku N, Maruyama I, Osame M. Predictive value of serial platelet count and VEGF determination for the management of DIC in the Crow-Fukase (POEMS) syndrome. Intern Med 2003;42:1240-3. DOI PubMed

7. Garcia J, Hurwitz HI, Sandler AB, et al. Bevacizumab $\left(\right.$ Avastin $^{\circledR}$ ) in cancer treatment: A review of 15 years of clinical experience and future outlook. Cancer Treat Rev 2020;86:102017. DOI PubMed

8. Badros A, Porter N, Zimrin A. Bevacizumab therapy for POEMS syndrome. Blood 2005;106:1135. DOI PubMed 
9. Dietrich PY, Duchosal MA. Bevacizumab therapy before autologous stem-cell transplantation for POEMS syndrome. Ann Oncol 2008;19:595. DOI PubMed

10. Straume O, Bergheim J, Ernst P. Bevacizumab therapy for POEMS syndrome. Blood 2006;107:4972-3; author reply 4973. DOI PubMed

11. Samaras P, Bauer S, Stenner-Liewen F, et al. Treatment of POEMS syndrome with bevacizumab. Haematologica 2007;92:1438-9. DOI PubMed

12. Ohwada C, Nakaseko C, Sakai S, et al. Successful combination treatment with bevacizumab, thalidomide and autologous PBSC for severe POEMS syndrome. Bone Marrow Transplant 2009;43:739-40. DOI PubMed

13. Zagouri F, Kastritis E, Gavriatopoulou M, et al. Lenalidomide in patients with POEMS syndrome: a systematic review and pooled analysis. Leuk Lymphoma 2014;55:2018-23. DOI PubMed 